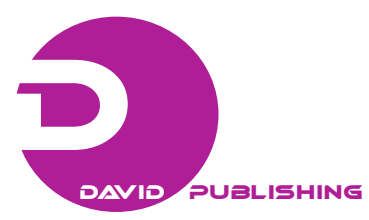

\title{
Mechanical Properties of Graphene within the Framework of Gradient Theory of Adhesion
}

\author{
Petr Anatolevich Belov \\ Engineering Research and Education Center, Bauman Moscow State Technical University, Moscow 117465, Russia
}

\begin{abstract}
The gradient model of two-dimensional defectless medium is formulated. A graphene sheet is examined as an example of such two-dimensional medium. The problem statement of a graphene sheet deforming in its plane and the bending problem are examined. It is ascertained that the statement of the first problem is equivalent to the flat problem statement of Toupin gradient theory. The statement of the bending problem is equivalent to the plate bending theory of Timoshenko with certain reserves. The characteristic feature of both statements is the fact that the mechanical properties of the sheet of graphene are not defined by "volumetric" moduli but by adhesive ones which have different physical dimension that coincides with the dimension of the corresponding stiffness of classical and nonclassical plates.
\end{abstract}

Key words: Gradient theories of elasticity, ideal adhesion, gradient adhesion, mechanical properties of graphene, nonclassical moduli.

\section{Introduction}

The generalization of Mindlin's model built [1] is under study. Unlike the "classical” models of Mindlin [2] and Toupin [3], its generalization takes into consideration not only the curvatures connected with the gradient of the free distortion in the volumetric density of potential energy but also the curvatures connected with the gradient of the restricted distortion, as well as their interaction. Another difference is considering the generalized model of the surface potential energies (the energy of adhesion interactions)

$L=A-\frac{1}{2} \iiint\left[C_{i j m n}^{11} D_{i j}^{1} D_{m n}^{1}+2 C_{i j m n}^{12} D_{i j}^{1} D_{m n}^{2}+C_{i j m n}^{22} D_{i j}^{2} D_{m n}^{2}\right.$

$\left.+C_{i j k m n l}^{11} D_{i j k}^{1} D_{m n l}^{1}+2 C_{i j k m n l}^{12} D_{i j k}^{1} D_{m n l}^{2}+C_{i j k m n}^{22} D_{i j k}^{2} D_{m n l}^{2}\right] d V$

$-\frac{1}{2} \oiint\left[A_{i j m n}^{11} D_{i j}^{1} D_{m n}^{1}+2 A_{i j m n}^{12} D_{i j}^{1} D_{m n}^{2}+A_{i j m n}^{22} D_{i j}^{2} D_{m n}^{2}\right.$

$\left.+A_{i j k m n l}^{11} D_{i j k}^{1} D_{m n l}^{1}+2 A_{i j k m n l}^{12} D_{i j k}^{1} D_{m n l}^{2}+A_{i j k m n}^{22} D_{i j k}^{2} D_{m n l}^{2}\right] d F$

$-\sum \oint U_{s} d s-\sum U_{p}$

Corresponding author: Petr Anatolevich Belov, Ph.D., research fields: mathematical models of properties of composite materials and technological processes, nanomechanics, gradient theories of elasticity, adhesion theories. E-mail: BelovPA@yandex.ru. in the Lagrangian, the surface edges energy $U_{s}$ and the energy of specific points of the surface edges $U_{p}$. Particularly, the Lagrangian of the generalized model can be presented the above Eq. (1).

The kinematic variables of the Lagrangian are:

(1) the continuous part of the displacement vector $R_{i}$;

(2) the distortions of two types $D_{i j}^{1}, D_{i j}^{2}$ (restricted and free distortions);

(3) the curvatures of two types $D_{i j k}^{1}, D_{i j k}^{2}$ (the gradients of the corresponding distortions).

Between these kinematic variables, there are restrictions defining the kinematic model of such medium:

$$
D_{i j}^{1}=R_{i, j} ; \quad D_{i j k}^{1}=R_{i, j k} ; \quad D_{i j k}^{2}=D_{i j, k}^{2}
$$

The tensors of moduli $C_{i j m n}^{p q}$ and $C_{i j k m n l}^{p q}$ define the mechanical properties of medium in volume and the tensors $A_{i j m n}^{p q}$ and $A_{i j k m n l}^{p q}$ define them on the medium surface.

This model demonstrates some new qualitative results which are impossible to be obtained in the frames of simpler models. One of such results is studied in this work, and namely the opportunity to 
explain the mechanical properties of two-dimensional medium and to make applied theories of bending and deforming in the graphene sheet plane as a two-dimensional medium.

Actually, the Lagrangians of both the classical mechanics of continuous medium and well-known gradient models of Mindlin and Toupin contain the potential energy defined only through the volumetric density of the potential energy. Formally, the Lagrangians of these models cannot be applied to the two-dimensional medium. This statement follows from the fact that in these models the potential energy of the zero volume medium equals null. In these models, all plate theories are formulated as the three-dimensional body models with a small, when compared to others, size in the third direction. Nevertheless, the same substantial mistake exists in the plate theory of these models, i.e., the plate of zero volume (due to its zero thickness) will have zero potential energy. One cannot consider the example of a graphene sheet as a volumetric structure having the thickness of about a carbon atom diameter as correct [4]. Actually, let us examine such a volumetric structure as a graphite plate consisting of parallel graphene sheets. It is quite obvious that "volumetric" properties of such a structure are defined by interatomic interactions of "long graphite" links of the carbon atoms of the adjacent graphene sheets. The interpolation of the properties of a multilayer and even two-layer graphite plate to the properties of an isolated graphene sheet is unacceptable. Thereby, it is reasonable to try to describe the mechanical properties of graphene within the theory, the Lagrangian of which contains the surface potential energy as well as the volumetric one. In the work [5], the variant of the theory of thin films with face adhesive properties was examined. However, the bending equation degenerated into a second-degree equation in the extreme case which is considered here at a zero volume film. It was defined by the fact that then only ideal, not gradient face adhesive properties were taken into consideration. The definition of a more general theory [1] allows now to turn to this problem and to formulate a non-degenerated case.

\section{Variational Method of Problem Statement}

In case of Lagrangian Eq. (1), if the medium volume equals null, the Lagrangian becomes a nontrivial specifically simple type:

$$
\begin{aligned}
L & =A-\frac{1}{2} \oiint\left[A_{i j m n}^{11} D_{i j}^{1} D_{m n}^{1}+2 A_{i j m n}^{12} D_{i j}^{1} D_{m n}^{2}\right. \\
& +A_{i j m n}^{22} D_{i j}^{2} D_{m n}^{2}+A_{i j k m n l}^{11} D_{i j k}^{1} D_{m n l}^{1} \\
& \left.+2 A_{i j k m n l}^{12} D_{i j k}^{1} D_{m n l}^{2}+A_{i j k m n l}^{22} D_{i j k}^{2} D_{m n l}^{2}\right] d F
\end{aligned}
$$

If graphene is considered as an ideal two-dimensional periodical structure, then we should put aside all terms containing the free distortion tensor $D_{i j}^{2}$ from Eq. (3) due to the fact that this tensor determines the defectness of the medium under study. The Lagrangian becomes as follows:

$$
L=A-\frac{1}{2} \oiint\left[A_{i j m n}^{11} D_{i j}^{1} D_{m n}^{1}+A_{i j k m n l}^{11} D_{i j k}^{1} D_{m n l}^{1}\right] d F
$$

Besides as graphene is a two-dimensional structure, the surface density of potential energy should not depend on normal derivatives of displacements. In connection with this fact, we should demand that the tensors of adhesive moduli have the following properties:

$$
\begin{gathered}
A_{i j m n}^{11} n_{j}=A_{i j m n}^{11} n_{n}=0 \\
A_{i j k m n l}^{11} n_{j}=A_{i j k m n l}^{11} n_{k}=A_{i j k m n l}^{11} n_{n}=A_{i j k m n l}^{11} n_{l}=0
\end{gathered}
$$

Here, $n_{j}$ is a unit normal vector of the graphene sheet plane. To simplify the task, let us accept the idea that the mechanical properties are isotropic in the graphene sheet plane. The result of Eqs. (4) and (5) is the next structure of adhesive tensors, simpler in comparison with Eq. (1):

$$
A_{i j m n}^{11}=\lambda^{F} \delta_{i j}^{*} \delta_{m n}^{*}+\mu^{F}\left(\delta_{i m}^{*} \delta_{j n}^{*}+\delta_{i n}^{*} \delta_{j m}^{*}\right)+\delta^{F} n_{i} n_{m} \delta_{j n}^{*}
$$




$$
\begin{aligned}
& A_{i j k m n l}^{11} \\
& =A_{1}^{11}\left(\delta_{i j}^{*} \delta_{k m}^{*} \delta_{n l}^{*}+\delta_{m n}^{*} \delta_{l i}^{*} \delta_{j k}^{*}+\delta_{i j}^{*} \delta_{l m}^{*} \delta_{n k}^{*}+\delta_{m n}^{*} \delta_{k i}^{*} \delta_{j l}^{*}\right. \\
& +\delta_{i k}^{*} \delta_{j m}^{*} \delta_{n l}^{*}+\delta_{m l}^{*} \delta_{m i}^{*} \delta_{j k}^{*}+\delta_{i l}^{*} \delta_{j m}^{*} \delta_{n k}^{*}+\delta_{m k}^{*} \delta_{m i}^{*} \delta_{j l}^{*} \\
& \left.+\delta_{i j}^{*} \delta_{k l}^{*} \delta_{m n}^{*}+\delta_{i k}^{*} \delta_{j n}^{*} \delta_{m l}^{*}+\delta_{i l}^{*} \delta_{j n}^{*} \delta_{m k}^{*}+\delta_{i n}^{*} \delta_{l k}^{*} \delta_{j m}^{*}\right) \\
& +A_{2}^{11} \delta_{i m}^{*}\left(\delta_{j k}^{*} \delta_{m l}^{*}+\delta_{j n}^{*} \delta_{k l}^{*}+\delta_{j l}^{*} \delta_{k n}^{*}\right) \\
& +A_{3}^{11} n_{i} n_{m}\left(\delta_{j k}^{*} \delta_{n l}^{*}+\delta_{j n}^{*} \delta_{k l}^{*}+\delta_{j l}^{*} \delta_{k n}^{*}\right)
\end{aligned}
$$

Here, $\quad \delta_{i j}^{*}=\left(\delta_{i j}-n_{i} n_{j}\right)$ is a "flat" tensor of Kronecker. The expanded structure of the potential energy becomes as follows:

$$
\begin{aligned}
U_{F} & =\frac{1}{2}\left\{\lambda^{F} r_{i, j} r_{m, n}+\mu^{F}\left(\delta_{j n}^{*} r_{m, j} r_{m, n}+r_{n, j} r_{j, n}\right)\right. \\
& +4 A_{1}^{11} \delta_{m n}^{*}\left(r_{i, j i} r_{j, m n}+r_{i, i m} r_{j, j n}+r_{i, j m} r_{j, i n}\right) \\
& +A_{2}^{11} r_{i, j k} r_{i, n l}\left(\delta_{j k}^{*} \delta_{n l}^{*}+\delta_{j n}^{*} \delta_{k l}^{*}+\delta_{j l}^{*} \delta_{k n}^{*}\right) \\
& +\delta^{F} \delta_{j n}^{*} R_{, j} R_{, n} \\
& \left.+A_{3}^{11} R_{, j k} R_{, n l}\left(\delta_{j k}^{*} \delta_{n l}^{*}+\delta_{j n}^{*} \delta_{k l}^{*}+\delta_{j l}^{*} \delta_{k n}^{*}\right)\right\}
\end{aligned}
$$

Here, the displacement vector $R_{i}=r_{i}+R n_{i}$ is presented as an expansion into a deflection $R$ and a projection on the plane of sheet $r_{i}$. In this case, as opposed to the classical plate's theory, the graphene sheet bending problem is always separated from the deforming problem in its plane. Let us examine these two problems separately.

\section{The Mechanical Properties of Graphene While Deforming in Its Plane}

In accordance with the definition of the potential energy Eq. (8) expressing of a graphene sheet while deforming in its plane, the deflections equal null.
Then the Lagrangian becomes as follows:

$$
\begin{aligned}
L & =A-\frac{1}{2} \iint\left\{\lambda^{F} r_{i, i} r_{m, m}+\mu^{F} \delta_{j n}^{*} r_{m, j} r_{m, n}\right. \\
& +\mu^{F} r_{n, j} r_{j, n} \\
& +4 A_{1}^{11} \delta_{m n}^{*}\left(r_{i, i j} r_{j, m n}+r_{i, i m} r_{j, j n}+r_{i, j m} r_{j, i n}\right) \\
& \left.+A_{2}^{11} r_{i, j k} r_{i, n l}\left(\delta_{j k}^{*} \delta_{n l}^{*}+\delta_{j n}^{*} \delta_{k l}^{*}+\delta_{j l}^{*} \delta_{k n}^{*}\right)\right\} d F
\end{aligned}
$$

We can define the force factors using the Green's formulae:

$$
\begin{aligned}
& \sigma_{i j}^{11}=\frac{\partial U_{F}}{\partial r_{i, j}}=\lambda^{F} \delta_{i j}^{*} r_{m, m}+\mu^{F}\left(\delta_{j n}^{*} r_{i, n}+\delta_{i n}^{*} r_{j, n}\right) \\
& m_{i j k}^{11}=\frac{\partial U_{F}}{\partial r_{i, j k}}=4 A_{1}^{11}\left(\delta_{i j}^{*} \delta_{m n}^{*} r_{k, m n}+\delta_{i j}^{*} \delta_{k n}^{*} r_{p, p n}\right. \\
& \left.+\delta_{k n}^{*} r_{j, i n}\right)+A_{2}^{11} r_{i, n l}\left(\delta_{j k}^{*} \delta_{n l}^{*}+\delta_{j n}^{*} \delta_{k l}^{*}+\delta_{j l}^{*} \delta_{k n}^{*}\right)
\end{aligned}
$$

The variation equation in the force factors is:

$$
\begin{aligned}
& \delta L=\iint\left(\sigma_{i j, j}^{11}-m_{i j k, j k}^{11}+P_{i}^{F}\right) \delta r_{i} d F \\
& \quad+\oint\left\{-\left(m_{i j k}^{11} v_{j} v_{k}\right) \delta\left(r_{i, p} v_{p}\right)+\left(P_{i}^{s}-\sigma_{i j}^{11} v_{j}\right.\right. \\
& \left.\left.\quad+m_{i j k, k}^{11} v_{j}+\left[\left(m_{i j k}^{11} s_{j} v_{k}\right) s_{p}\right]_{, p}\right) \delta r_{i}\right\} d s \\
& \quad-\sum\left(m_{i j k}^{11} s_{j} v_{k}\right) \delta r_{i}=0
\end{aligned}
$$

Here, we use curvilinear orthogonal coordinates with unit vectors $s_{i}$ and $v_{i}$, connected with the graphene sheet contour, $P_{i}^{F}$ is $2 \mathrm{D}$ external loads, tangent to the sheet plane. $P_{i}^{s}$ is contour external loads in the sheet plane.

The variation equation in displacements is:

$$
\begin{aligned}
\delta L & =\delta A+\iint\left\{\mu^{F} \nabla^{2} r_{i}+\left(\mu^{F}+\lambda^{F}\right) r_{k, k i}-12 A_{1}^{11} \nabla^{2} r_{k, k i}-3 A_{2}^{11} \nabla^{2} \nabla^{2} r_{i}\right\} \delta r_{i} d F \\
& -\oint\left\{\left[4 A_{1}^{11}\left(r_{k, k i}+r_{k, k m} v_{i} v_{m}+r_{j, i m} v_{j} v_{m}\right)+A_{2}^{11} r_{i, k m}\left(s_{m} s_{k}+3 v_{m} v_{k}\right)\right] \delta\left(r_{i, p} v_{p}\right)\right\} d s \\
& -\oint\left\{\left[\lambda^{F} r_{m, m} v_{i}+\mu^{F} r_{i, j} v_{j}+\mu^{F} r_{j, i} v_{j}\right]\right. \\
& -\left[4 A_{1}^{11}\left(r_{k, k i j} v_{j}+\nabla^{2} r_{k, k} v_{i}+\nabla^{2} r_{j, i} v_{j}\right)+3 A_{2}^{11} \nabla^{2} r_{i, j} v_{j}\right] \\
& \left.-\left[4 A_{1}^{11}\left(r_{k, k m p} s_{i} v_{m} s_{p}+r_{j, i m p} s_{j} v_{m} s_{p}\right)+2 A_{2}^{11} r_{i, k m p} s_{k} v_{m} s_{p}\right]\right\} \delta r_{i} d s \\
& -\sum\left[4 A_{1}^{11}\left(r_{k, k m} s_{i} v_{m}+r_{j, i m} s_{j} v_{m}\right)+2 A_{2}^{11} r_{i, k m} s_{k} v_{m}\right] \delta r_{i}=0
\end{aligned}
$$


Let us introduce the following denominations: a derivative along the contour: $r_{i, k} s_{k}=r_{i}^{\prime}$; normal contour derivative: $r_{i, k} v_{k}=\dot{r}_{i}$. Then the variation equation in displacements for the rectangular contour becomes Eq. (13).

Here, $\nabla^{2}(\ldots)=(\ldots)_{, i j} \delta_{i j}^{*}$ is the Laplace flat operator.

$$
\begin{aligned}
\delta L & =\iint\left\{\mu^{F} \nabla^{2} r_{i}+\left(\mu^{F}+\lambda^{F}\right) r_{k, k i}-12 A_{1}^{11} \nabla^{2} r_{k, k i}-3 A_{2}^{11} \nabla^{2} \nabla^{2} r_{i}+P_{i}^{F}\right\} \delta r_{i} d F \\
& -\oint\left[4 A_{1}^{11}\left(r_{k, k i}+\dot{r}_{k, k} v_{i}+\dot{r}_{j, i} v_{j}\right)+A_{2}^{11}\left(r_{i}^{\prime \prime}+3 \ddot{r_{i}}\right)\right] \delta \dot{r}_{i} d s \\
& -\oint\left\{\left[\lambda^{F} r_{m, m} v_{i}+\mu^{F} \dot{r}_{i}+\mu^{F} r_{j, i} v_{j}\right]\right. \\
& -\left[4 A_{1}^{11}\left(\dot{r}_{k, k i}+\nabla^{2} r_{k, k} v_{i}+\nabla^{2} r_{j, i} v_{j}\right)+3 A_{2}^{11} \nabla^{2} \dot{r}_{i}\right] \\
& \left.-\left[4 A_{1}^{11}\left(\dot{r}_{k, k}^{\prime} s_{i}+\dot{r}_{j, i}^{\prime} s_{j}\right)+2 A_{2}^{11} \dot{r}_{i}^{\prime \prime}\right]-P_{i}^{S}\right\} \delta r_{i} d s \\
& -\sum\left[4 A_{1}^{11}\left(r_{k, k m} s_{i} v_{m}+r_{j, i m} s_{j} v_{m}\right)+2 A_{2}^{11} r_{i, k m} s_{k} v_{m}\right] \delta r_{i}=0
\end{aligned}
$$

Thereby, the graphene sheet deformation model is equivalent to the flat problem definition of Toupin gradient model but with other physical properties defined by other (adhesive) moduli tensors. The tensors of adhesive moduli have physical dimension which coincides with the dimension of the corresponding stiffness of classical and gradient theory plates.

\section{The Mechanical Properties of Graphene While Bending}

In accordance with the definition of the potential energy expressing of a graphene sheet Eq. (8) while deforming the sheet from its plane, the Lagrangian becomes as follows:

$$
L=A-\frac{1}{2} \iint\left\{\delta^{F} \delta_{p q}^{*} R_{, p} R_{, q}+A_{3}^{11}\left(\delta_{j k}^{*} \delta_{n l}^{*}+\delta_{j n}^{*} \delta_{k l}^{*}+\delta_{j l}^{*} \delta_{k n}^{*}\right) R_{, j k} R_{, n l}\right\} d F
$$

We can define the force factors using the Green's formulae:

$$
\begin{aligned}
& Q_{p}=\frac{\partial U_{F}}{\partial R_{, p}}=\delta^{F} \delta_{p q}^{*} R_{, q}=\left\{\begin{array}{l}
Q_{x}=\delta^{F} R_{, x} \\
Q_{y}=\delta^{F} R_{, y}
\end{array}\right. \\
& M_{j k}=\frac{\partial U_{F}}{\partial R_{, j k}}=A_{3}^{11}\left(\delta_{j k}^{*} \nabla^{2} R+\delta_{l j}^{*} \delta_{n k}^{*} R_{, n l}+\delta_{n j}^{*} \delta_{k l}^{*} R_{, n l}\right)=\left\{\begin{array}{l}
M_{x x}=A_{3}^{11}\left(3 R_{, x x}+R_{, y y}\right) \\
M_{x y}=A_{3}^{11} 2 R_{, x y} \\
M_{y x}=A_{3}^{11} 2 R_{, x y} \\
M_{y y}=A_{3}^{11}\left(R_{, x x}+3 R_{, y y}\right)
\end{array}\right.
\end{aligned}
$$

The variation equation in the force factors is:

$$
\begin{aligned}
\delta L & =\delta A-\iint\left\{Q_{j} \delta R_{, j}+M_{j k} \delta R_{, j k}\right\} d F \\
& =\iint\left(Q_{j, j}-M_{j k, j k}+P^{F}\right) \delta R d F \\
& +\oint\left\{-\left(M_{j k} v_{j} v_{k}\right) \delta\left(R_{, i} v_{i}\right)+\left(P^{F}-Q_{j} v_{j}-\left[\left(M_{j k} s_{j} v_{k}\right) s_{i}\right]_{, i}+M_{j k, k} v_{j}\right) \delta R\right\} d s \\
& -\sum\left(M_{j k} s_{j} v_{k}\right) \delta R=0
\end{aligned}
$$

The natural boundary conditions can be compared with the boundary value problems definition in the classical plate theory. Just as in the classical plate theory, a demand for the torsion moment 
continuity $\left(M_{j k} s_{j} v_{k}\right)$ appears while crossing the contour specific point (an integrated item of the variation equation). The possible work of the bending moment on the turning angle $\left(M_{j k} v_{j} v_{k}\right) \delta\left(R_{, i} v_{i}\right)$ also produces the classical pair of boundary conditions: either one should specify the turning angle or the bending moment should equal null. Here we cannot but mention the fact that in the frames of the graphene sheet bending theory, there is no possibility to specify the bending moment on the contour that will not equal null. Another pair of the natural boundary conditions is connected with the possible work of the shear force at various deflections $\left(P^{F}-Q_{j} v_{j}-\left[\left(M_{j k} s_{j} v_{k}\right) s_{i}\right]_{, i}+M_{j k, k} v_{j}\right) \delta R$. In this case, just as in the classical plate theory, it is not possible to formulate the boundary condition for Saint-Venant's shear force $Q_{j} v_{j}$, so we have to introduce the definition of Kirchhoff's shear force. Simultaneously, as opposed to the classical plate theory, it is modified not only by the torsion moment contour derivative $\left[\left(M_{j k} s_{j} v_{k}\right) s_{i}\right]_{, i}$ but also by two additional items. The first item is the torsion moment contour derivative $M_{j m, n} v_{j} s_{m} s_{n}$, and the second item is the bending moment normal derivative $M_{j m, n} v_{j} v_{m} v_{n}$. Being summed up, they give the "flat" divergence $M_{j k, k} v_{j}$.

The variation equation in the deflection is:

$$
\begin{aligned}
\delta L & =\iint\left[\delta^{F} \nabla^{2} R-3 A_{3}^{11} \nabla^{2} \nabla^{2} R+P^{F}\right] \delta R d F \\
& +\oint\left\{-A_{3}^{11}\left(R^{\prime \prime}+3 \ddot{R}\right) \delta \dot{R}+\left[P^{s}-\delta^{F} \dot{R}\right.\right. \\
& \left.\left.+3 A_{3}^{11} \dddot{R}+5 A_{3}^{11} \dot{R}^{\prime \prime}\right] \delta R\right\} d s \\
& -\sum 2 A_{3}^{11} \dot{R}^{\prime} \delta R=0
\end{aligned}
$$

Just as in the theory of thin films with an ideal face adhesion stated in Ref. [5], the resolving equation of the graphene sheet bending theory contains not only the biharmonic operator but also the harmonic one, i.e., the differential operator of this equation has the same structure as in the Timoshenko's theory. The operators' factors $\delta^{F}, A_{3}^{11}$ are adhesive moduli which in the physical dimension coincide with the
Timoshenko plate theory stiffness $G h$ and $E h^{3} / 12\left(1-v^{2}\right)$.

\section{Conclusions}

The applied theories of a graphene sheet bending and deforming it in its plane formulated in this work offer possibilities to study the mechanical properties of $2 \mathrm{D}$ media, to set and solve test problems the solutions of which can be tested experimentally. In particular, the bending problem makes it possible to reduce the graphene mechanical properties to two non-classical modules $\delta^{F}$ and $A_{3}^{11} ;$ and correspondingly the plane deforming problem reduces them to four: $\lambda^{F}, \mu^{F}, A_{1}^{11}$ and $A_{2}^{11}$. We should pay our attention to the fact that the model formulated in this work can be generalized as any other theories.

Firstly, in this work the graphene theory bases on the hypothesis that there are no adhesion interactions between distortions $R_{i, j}$ and curvatures $R_{m, n l}$ which is equivalent to the fact that the moduli of the fifth rank tensor $A_{i j m n l}^{11}$ equal null. We can insist with certainty that taking into account such interaction $\left(A_{i j m n l}^{11} R_{i, j} R_{m, n l} / 2\right)$ in adhesion potential energy in the general case for the problems of bending and deforming in plane will lead to the coherence regeneration of the equilibrium equations' system.

Secondly, the generalization should engage the formulating of the potential energy of the surface edges as well. In applied graphene theory, this will also lead to the re-formulating and coherence of the boundary conditions.

Thirdly, the qualitative difference of mechanical properties of $3 \mathrm{D}$ and $2 \mathrm{D}$ media, stated in this work, demands the answer to the question: "Are such nanostructures as graphene, nanotubes and fullerenes truly two-dimensional structures or shall we simulate them as 'edge' systems?" As we stated in Ref. [1], the edges' mechanical properties differ from the surface properties in the way the surface properties differ from the properties of $3 \mathrm{D}$ media. 


\section{Acknowledgments}

Thanks go to the Translators Tatiana Vizavitska and Anush Melikyan who translate this article into English.

\section{References}

[1] P.A. Belov, The theory of continuum with conserved dislocations: Generalization of Mindlin theory, Composites and Nanostructures 3 (1) (2011) 24-38.

[2] R.D. Mindlin, Micro-structure in linear elasticity,
Archive of Rational Mechanics and Analysis 1 (1964) 51-78.

[3] R.A. Toupin, Elastic materials with couple-stresses, Archive of Rational Mechanics and Analysis 2 (1964) 85-112.

[4] A.K. Geim, K.S. Novoselov, D. Jiang, F. Schedin, T.J. Booth, V.V. Khotkevich, et al., Two-dimensional atomic crystals, Proceedings of the National Academy of Sciences 102 (30) (2005) 10451-10453.

[5] P.A. Belov, S.A. Lurie, The theory of ideal adhesion interactions, The Mechanics of Composite Materials and Designs 14 (3) (2007) 519-536. 